\title{
Valores sanguíneos del loro hablador chaqueño (Amazona aestiva xanthoptery $x$ ) adulto, en cautiverio en el Centro de Rescate ".La Esmeralda“ de la provincia de Santa Fe, Argentina.
}

\section{Blood values of the Chaco chatter parrot (Amazona aestiva xanthopteryx) adult, in captivity at La Esmeralda Rescue Center in the province of Santa Fe, Argentina}

\section{Valores sanguíneos do papagaio falante do chaco (Amazona aestiva xanthopteryx) adulto, em cativeiro no Centro de Resgate "La Esmeralda" da província de Santa Fé, Argentina}

\author{
Antonio Sciabarrasi ${ }^{1-2}$; Marcelo Ruíz ${ }^{3}$; Pablo Siroski ${ }^{4}$.
}

1. Cátedra de Zoología, Diversidad y Ambiente, Facultad de Ciencias Veterinarias, Universidad Nacional del Litoral. Kreder 2805, (3080) Esperanza, Santa Fe, Argentina.

2. Estación Biológica La Esmeralda, (3000) Santa Fe, Argentina.

3. Laboratorio de Análisis Clínico FCV-UNL. mruiz@fcv.unl.edu.ar

4. Centro de Medicina Comparada-Icivet Litoral, FCV-UNL. psiroski@fcv.unl.edu.ar

Recibido: 6 de febrero de 2019 Corregido: 31 de mayo de 2019 Aceptado: 14 de junio de 2019

\begin{abstract}
Resumen: Amazona aestiva xanthopteryx conocido comúnmente como loro hablador chaqueño, amazona de alas amarillas o amazona argentino. Su población se distribuye en Argentina desde el distrito fitogeográfico de la Selva Pedemontana de la región fitogeográfica de las Yungas y las áreas próximas a los distritos del Chaco Occidental, Árido y Serrano. En ese país es uno de los loros más traficados ilegalmente. Los aspectos básicos de la patología clínica los cuales incluyen a la hematología han sido utilizados comúnmente en el diagnóstico de aves enfermas; sin embargo, es necesario conocer los parámetros fisiológicos teniendo en cuenta las variables pertinentes a cada región y sus respectivas condiciones. El objetivo del presente estudio fue describir los valores obtenidos para eritrocitos, Hematocrito (Hto), Hemoglobina (Hb), Volumen Corpuscular Medio (VCM), Hemoglobina Corpuscular Media (HCM), Concentración de Hemoglobina Corpuscular Media (CHCM), conteo absoluto y diferencial de leucocitos y conteo absoluto de plaquetas de una población aparentemente sana de 27 individuos cautivos de ésta subespecie, alojados en el Centro de Rescate, Rehabilitación y Reubicación de Fauna "La Esmeralda" de la Provincia de Santa Fe en Argentina. Los datos obtenidos fueron tabulados y analizados mediante estadística descriptiva. Los resultados para los distintos parámetros fueron en su mayoría similares a los reportados para otras aves Psittaciformes, con mínimas diferencias asociadas a las distintas condiciones ambientales y de manejo de cada estudio. Los valores obtenidos del loro hablador del chaco permitirán ser una guía para la aplicación en la clínica y programas de rescate, conservación.
\end{abstract}

Palabras clave: Amazona aestiva xanthopteryx, hematología, loro, Psittaciformes.

Autor para correspondencia: asciabarrasi@fcv.unl.edu.ar 


\begin{abstract}
Amazona aestiva xanthopteryx commonly known as Chaco chatter parrot, yellow-winged amazon or Argentine amazon. Its population is distributed in Argentina from the phytogeographic district of the Selva Pedemontana of the Yungas phytogeographic region and the areas near the districts of Western Chaco, Arid and Serrano. In that country it is one of the parrots most illegally trafficked. The basic aspects of clinical pathology, which include hematology, have been commonly used in the diagnosis of diseased birds; however, it is necessary to know the physiological parameters taking into account the variables relevant to each region and their respective conditions. The aim of the present study was to describe the values obtained for erythrocytes, Hematocrit (Hct), Hemoglobin ( $\mathrm{Hb}$ ), Mean Corpuscular Volume (MCV), Middle Corpuscular Hemoglobin (MCH), Concentration of Middle Corpuscular Hemoglobin (MCH), absolute and differential counting of leukocytes and absolute platelet count of an apparently healthy population of 27 captive individuals belonging to this subspecies, located in the Center of Rescue, Rehabilitation and Relocation of La Esmeralda Fauna of the Province of Santa Fe in Argentina. The data obtained were tabulated and analyzed by descriptive statistics. The results for the different parameters were mostly similar to those reported for other Psittaciformes birds, with minimal differences associated with the different environmental and management conditions of each study. The values obtained from the talking parrot of the Chaco will be a guide for the application in the clinic and rescue programs, conservation.
\end{abstract}

Keywords: Amazona aestiva xanthopteryx, hematology, parrot, Psittaciformes.

Resumo: Amazona aestiva xanthopteryx, comumente conhecido como papagaio falante do chaco, Amazonas de asa amarela, Amazonas argentino. Na Argentina, sua população está distribuída desde o distrito fitogeográfico da Floresta Pedemontana da região fitogeográfica dos Yungas e das áreas próximas aos distritos do Chaco Ocidental, Árido e Serrano. Nesse país, esta espécie de papagaios é uma das mais traficadas ilegalmente. Os aspectos básicos da patologia clínica, que incluem hematologia, têm sido comumente usados no diagnóstico de aves doentes; no entanto, é necessário conhecer os parâmetros fisiológicos, considerando as variáveis relevantes para cada região e suas respectivas condições. O objetivo do presente estudo foi descrever os valores obtidos para eritrócitos, Hematócrito (Hto), Hemoglobina (Hb), Volume Corpuscular Médio (VCM), Hemoglobina Corpuscular Média (HCM), Concentração Média de Hemoglobina Corpuscular (CHCM), contagem absoluta e diferencial de leucócitos e contagem absoluta de plaquetas de uma população aparentemente saudável de 27 indivíduos em cativeiro dessa subespécie, alojados no Centro de Recuperação, Reabilitação e Destinação da fauna La Esmeralda localizada na Província de Santa Fé, Argentina. Os dados obtidos foram tabulados e analisados por estatística descritiva. Os resultados para os diferentes parâmetros foram semelhantes aos relatados para outras aves psitaciformes, com diferenças mínimas associadas às distintas condições ambientais e de manejo de cada estudo. Os valores obtidos com o papagaio falante do chaco permitirão ser um guia para a aplicação na clínica e nos programas de resgate e conservação.

Palavras-chave: Amazona aestiva xanthopteryx, hematologia, papagaio, Psittaciformes

\title{
Introducción
}

El loro hablador (Amazona aestiva) es una de las especies de amazonas más extendidas por Sudamérica; habita en selvas de galería desde el Centro de Bolivia y Brasil, y partes del norte de Paraguay y Argentina, con un tamaño corporal promedio es de $36 \mathrm{~cm}$, llegando a pesar entre 350-450 g. Es de color verde, frente y bridas azuladas, garganta, mejilla y píleo amarillos. La cabeza es de color verde, azul y amarillo. El borde de las alas es rojo o amarillo, con las coberteras alares rojas. Ojos de color naranja. El pico de color negruzco y las patas grises. Los ejemplares jóvenes presentan una coloración distinta a la de los adultos; donde se destaca el color gris oscuro de las pupilas hasta que las aves alcanzan la edad de un año. La especie no presenta dimorfismo sexual (Narosky \& Yzurieta 2010). 
La subespecie Amazona aestiva xanthopteryx (Fig. 1), es una de las 2 subespecies en que se divide Amazona aestiva, la misma se diferencia de ésta en que presenta las plumas cobertoras alares (zona de los hombros) de color amarillo de mayor extensión y siendo el color rojo de ésta región de menor distribución incluso pudiendo estar ausente (Fig. 1). El color general es de un verde más claro, con el amarillo, a veces blanco, de la cabeza más extendido y con distribución del azul de la frente reducida (Chébez 2008). El área núcleo de Amazona aestiva xanthopteryx es el distrito fitogeográfico de la Selva Pedemontana de la región fitogeográfica de las Yungas y las áreas próximas de la provincia Argentina de Chaco, distritos chaqueños Occidental, Árido, y Serrano. Al ser estas poblaciones muy inconstantes en sus caracteres morfológicos, ha impedido una diferenciación sistemática propia, por lo que se los asigna a Amazona aestiva xanthopteryx (Berkunsky et al. 2017).

La especie está protegida por el convenio CITES, constando en el Apéndice II (CITES 2017). La subespecie en cuestión es aún bastante difundida en algunas áreas de su distribución, aunque ha desaparecido o es rara en otras. Sus poblaciones están decreciendo debido principalmente a la destrucción de su hábitat para ser transformado en campos agrícola (Banchs et al. 2006). Otra amenaza creciente es la captura ilegal para el comercio como mascotas ya que en Argentina es el psitácido más apreciado como ave de jaula, por lo que es uno de los animales más comunes en el tráfico ilegal de fauna silvestre. Como consecuencia a lo antes dicho es que se han habilitado criaderos de esta especie en países de Europa, así como en Estados Unidos, Brasil y Argentina (Deem et al. 2005).

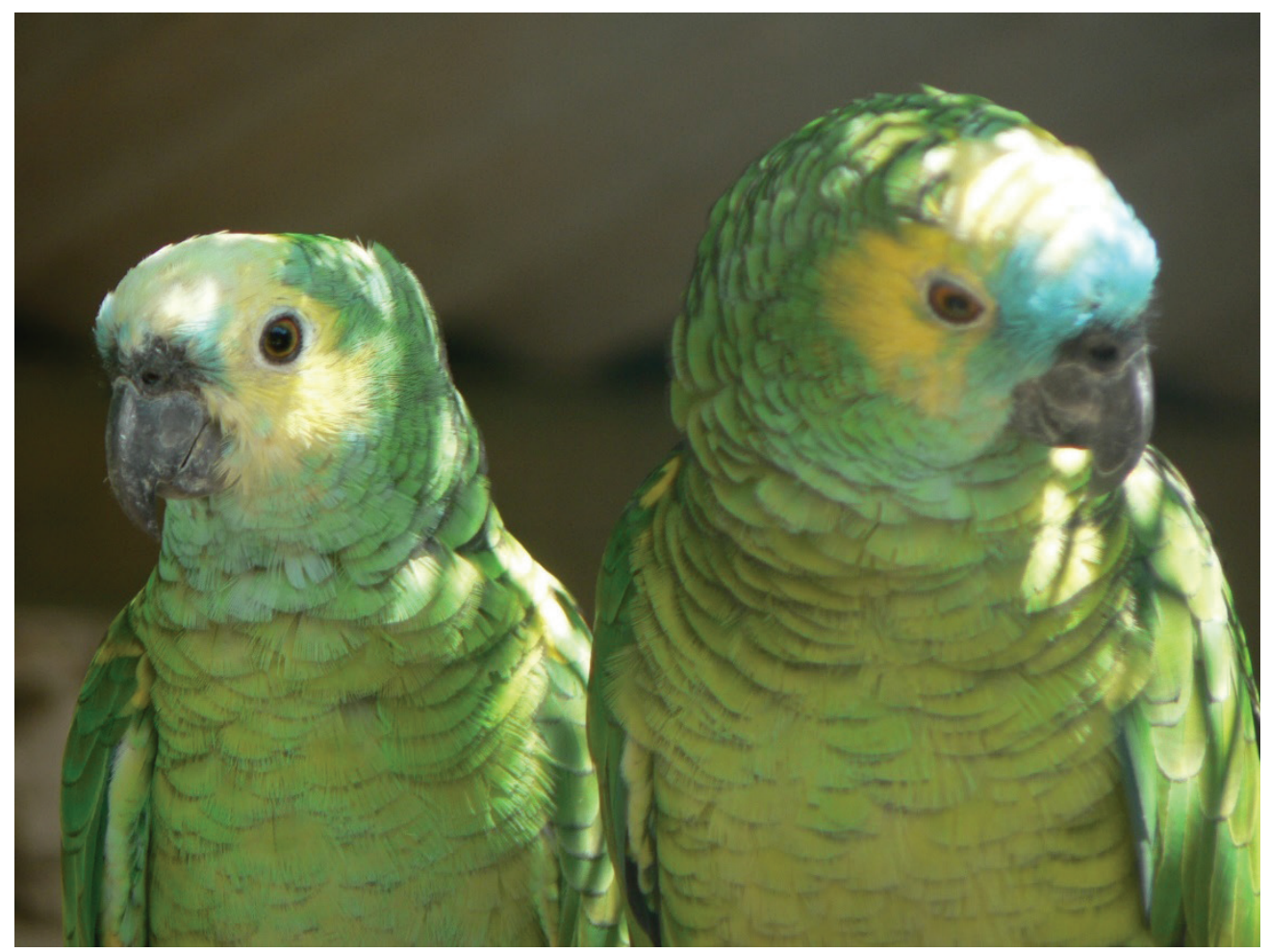

Figura $\mathbf{N}^{\circ}$ 1. Individuos de Amazona aestiva xanthopteryx, en la Estación de Rescate, Rehabilitación y Reubicación de Fauna "La Esmeralda" de la Provincia de Santa Fe en Argentina. 
Rev. Ciencias Veterinarias, Vol. 37, N² 2, [22-31], E-ISSN: 2215-4507, julio-diciembre, 2019

DOI: https://doi.org/10.15359/rcv.37-2.3

URL: http://www.revistas.una.ac.cr/index.php/veterinaria/index

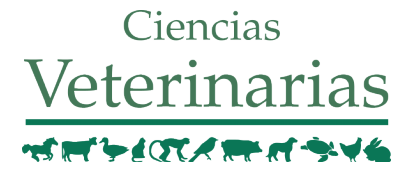

Sumado a lo antes dicho, éste aumento en el comercio legal e ilegal de estas aves, ha incrementado la frecuencia de estas en la clínica veterinaria, así como en centros de rescate y rehabilitación de fauna, a menudo por problemas relacionados con el desconocimiento de su ambiente, sus hábitos etológicos y alimenticios, lo cual ha despertado a su vez un gran interés por conocer su biología, etología y parámetros fisiológicos que ayuden a ofrecer una mejor atención a estos animales tanto en vida silvestre como en cautiverio (Paula et al. 2008).

Los aspectos básicos de la patología clínica, hematología y bioquímica sanguínea, han sido utilizados en el diagnóstico de aves enfermas (Paula et al. 2008). Sin embargo, la utilización e interpretación requieren poseer conocimiento previo de los parámetros fisiológicos básicos (Paula et al. 2008) de las diferentes especies en las respectivas regiones y condiciones (Cordeiro 2004; Valle et al. 2008) ya que puede servir como modelo para el estudio de la respuesta que tienen los animales de la misma especie o subespecie en su hábitat natural frente a un determinado desafío epidemiológico, así como facilitar un diagnóstico de enfermedad. La mayoría de las veces, las enfermedades de los animales silvestres en cautiverio son comparables con aquellas observadas en vida silvestre y, por lo tanto, determinados programas de conservación ambiental también pueden ser beneficiados con estos datos (Valle et al. 2008).

El objetivo del presente estudio fue describir los valores obtenidos de las variables sanguíneas del número absoluto de de Eritrocitos, Hematocrito (Hto), Hemoglobina (Hb), Volumen Corpuscular Medio (VCM), Hemoglobina Corpuscular Media (HCM), Concentración de Hemoglobina Corpuscular Media (CHCM), Conteo Diferenciales Absoluto y diferencial de Leucocitos y Recuento absoluto de Plaquetas de una población aparentemente sana de 27 individuos cautivos pertenecientes a la subespecie Amazona aestiva xanthopteryx, ubicados en la Estación de Rescate, Rehabilitación y Reubicación de Fauna "La Esmeralda” de la Provincia de Santa Fe en Argentina.

\section{Materiales y Métodos}

El presente trabajo se realizó en la Estación de Rescate, Rehabilitación y Reubicación de Fauna "La Esmeralda“ de la Provincia de Santa Fe en Argentina, durante el mes de agosto de 2018. Se utilizaron 27 individuos adultos, de los cuales eran 12 hembras y 15 machos. Las aves objeto de estudio, procedían de decomisos y entregas voluntarias realizadas en las provincias de Santa Fe y Mendoza. Al examen clínico las aves estaban aparentemente sanas y con pesos promedios de $380 \mathrm{~g}$ para las hembras y $430 \mathrm{~g}$ para los machos, sexado realizado por ADN en plumas (Fig.2). Recibían una dieta balanceada de frutas, vegetales y semillas, suministrada ad libitum renovada dos veces al día (mañana y tarde).

La captura fue mediante contención física (Soto et al. 2009), con una red con mango de metal a modo de copo; fueron manipuladas entre las 10:00 y las 11:00 de la mañana. Una vez capturada el ave, se procedió a su identificación por medio de anillos abiertos, luego se pesaron mediante una bolsa plástica, se realizaron las medidas morfométricas, se tomaron constantes fisiológicas (FR, FC y temperatura) y se realizó un examen clínico por sistemas en busca de posibles alteraciones con el fin de confirmar que se trataban

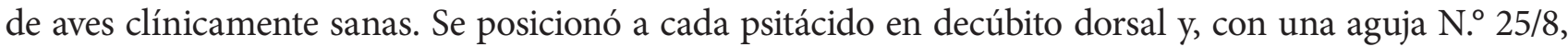
se extrajo $1 \mathrm{ml}$ total de sangre de las venas braquial (Fig.2). Parte de la sangre $(0.5 \mathrm{ml})$ se transfirió a un tubo con heparina de litio para el análisis hematológico (glóbulos rojos y blancos, hematocrito, y volumen corpuscular medio y con el $0.5 \mathrm{ml}$ restante se prepararon dos frotis por individuo, uno de ellos para 
la observación citomorfológica y, el último, para el recuento diferencial de leucocitos. Los recuentos de eritrocitos y leucocitos se realizaron mediante métodos manuales (cámara de Neubauer) con solución de Natt y Herrick. El hematocrito (Hto) por microhematocrito, la hemoglobina $(\mathrm{Hb})$ por colorimetría reacción de la cianometahemoglobina; el volumen corpuscular medio (VCM), la hemoglobina corpuscular media ( $\mathrm{HbCM}$ ) y la concentración de hemoglobina corpuscular media (CHCM) mediante las formulas expresadas por Meneses et al. 1993. El recuento diferencial de leucocitos fue por observación de frotis sanguíneos teñidos con el colorante de Wright aplicando la prueba de U de Mann Whitney (Sokal \& Rohlf 1995). Se realizó además conteo de plaquetas utilizando el hemocitómetro como lo indicó Ike et al. 2010. Los datos fueron tabulados y analizados mediante estadística descriptiva, utilizando el paquete estadístico de análisis de datos del programa Excel 2007 (Microsoft Corp.). Para determinar la influencia del sexo sobre los valores de hematología se utilizaron pruebas de t. Las pruebas estadísticas fueron realizadas utilizando el software $\mathrm{R}^{\oplus}$ (GNU projects).

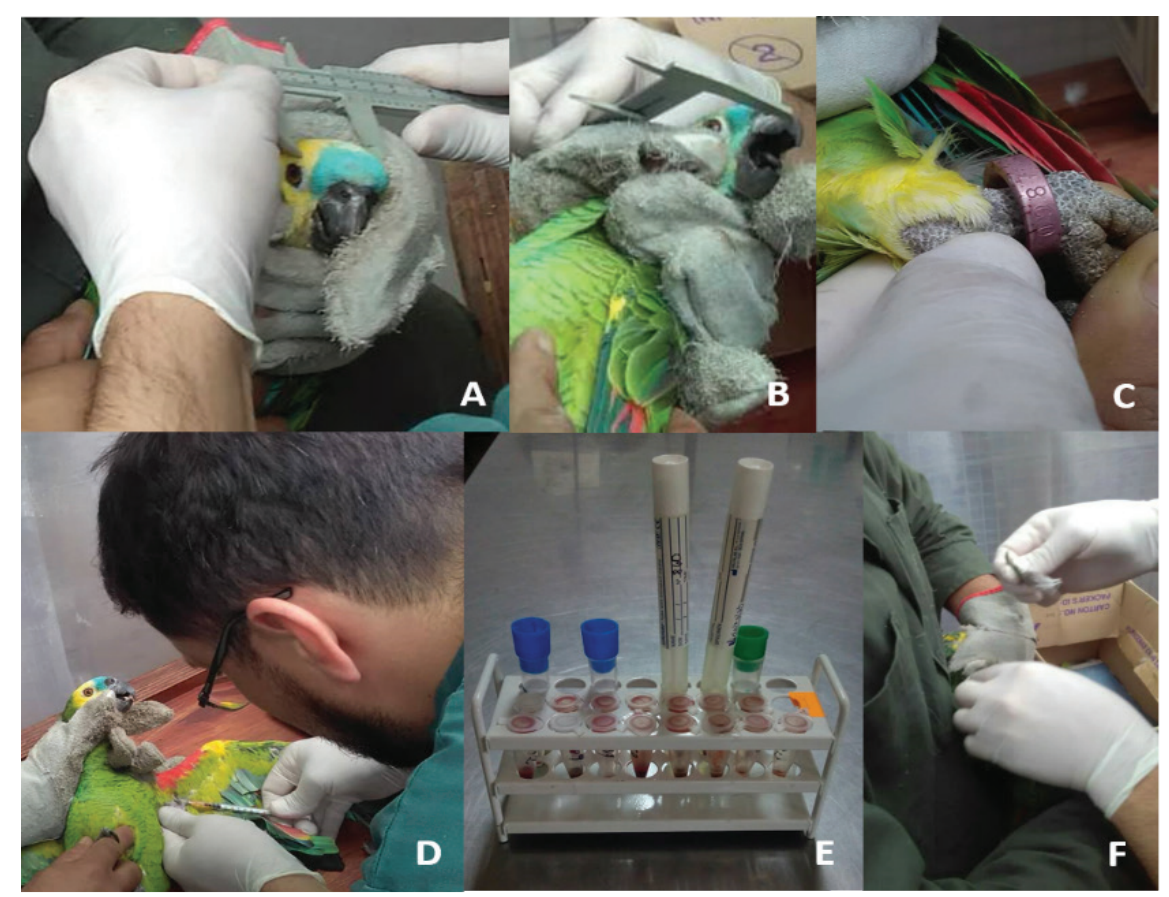

Figura $\mathbf{N}^{\circ}$ 2. Toma de muestras, morfometría y trazabilidad en Amazona aestiva xanthopteryx. A y B. Morfometría craneales. C. Anillado para trazabilidad. D. Extracción de sangre. E. Remisión de sangre con anticoagulante, suero e hisopados cloacales. F. Colecta de plumas enteras para sexado por ADN. En la Estación de Rescate, Rehabilitación y Reubicación de Fauna "La Esmeralda" de la Provincia de Santa Fe en Argentina.

\section{Resultados y Discusión}

La evaluación del hemograma ha sido una herramienta comúnmente utilizada en el diagnóstico de las enfermedades aviares, siendo los métodos manuales de laboratorio usados rutinariamente en 
humanos y mamíferos, los más utilizados para los estudios hematológicos en aves (Paula et al. 2008). Se determinaron valores medios para los parámetros hematológicos para machos y hembras del loro hablador chaqueño. Los resultados obtenidos para el análisis del Hto, Hb, se encuentran reportados en el cuadro 1. Comparando los resultados obtenidos con los reportados para la media del hematocrito $(45,23$ $\pm 5.40 \%)$ y la hemoglobina $(14,34 \pm 1.77 \mathrm{mg} / \mathrm{dl})$ se evidenció que fueron similares a los encontrados en la literatura consultada para otras aves Psittaciformes (Deem et al. 2005; Alvarado et al. 2008; Schmidt et al. 2009; Soto et al. 2009; Mérida \& Guerra 2011). Es de resaltar las diferencias obtenidas para el hematocrito y hemoglobina con el reporte realizado en la ciudad de São Paulo por Paula et al. (2008) para Amazona aestiva aestiva, obteniéndose en el presente estudio valores para la media superiores a los reportados por ellos (Hto $38.7 \pm 6.2 \%$; Hb $13.2 \pm 2.1 \mathrm{mg} / \mathrm{dl}$ ). Dicha diferencia puede ser debida por las posibles distintas condiciones de manejo y ambientales tales como el estrés causado en la manipulación, cautiverio (Paula et al. 2008). La excitación y el temor del ave en el momento de la extracción sanguínea pueden derivar en un aumento fisiológico en el recuento de glóbulos rojos, hematocrito, la hemoglobina e índices eritrocitarios y recuentos de leucocitos, por la liberación excesiva de corticoides endógenos (Franco et al. 2010). En comparación a lo reportado por Franco et al. (2010), se encontraron valores para la media inferiores a los reportados por ellos para el Hto de Amazona amazónica (50.4 \pm 1.4 ) y Amazona ochrocephala (53.8 \pm 1.2) en cautiverio y valores similares para la $\mathrm{Hb}$ con Amazona amazónica (16.7 \pm 0.47$)$ e inferiores para Amazona ochrocephala (17.9 \pm 0.39$)$. Dicha diferencia superior en estos valores en relación con otros reportes es atribuida por Franco et al. (2010) a distintos factores, como el lugar de procedencia, condiciones de confinamiento y factores ambientales.

Cuadro 1. Valores de hematocrito, hemoglobina en Amazona aestiva xanthopteryx $(\mathrm{n}=27)$ en la Estación de Rescate, Rehabilitación y Reubicación de Fauna "La Esmeralda“ de la Provincia de Santa Fe en Argentina.

\begin{tabular}{llll} 
Parámetro & \multicolumn{3}{c}{ Amazona aestiva xanthopteryx } \\
& Media \pm DE & Valor Min & Valor Max \\
\hline Hematocrito (\%) & $45,23 \pm 5.40$ & 43,23 & 48,63 \\
Hemoglobina (mg/dl) & $14,34 \pm 1.77$ & 13,44 & 15,12
\end{tabular}

DE: Desviación Estándar.

Sin embargo, no puede descartarse que un hematocrito elevado pueda ser causado por algún grado subclínico de deshidratación (Naidoo et al. 2008). Valores altos para el recuento de eritrocitos y hematocrito son influenciados por la altitud, disponibilidad de oxígeno y vuelos demandantes, por lo que estas variables deben considerarse al momento de transpolar los valores generados a otras poblaciones, en nuestro estudio los valores no fueron influenciados por dichas variables debido a la ubicación geográfica de los mismos como así también la ausencia del vuelo en todo el proceso.

Los valores que se obtuvieron (cuadro 2) para linfocitos son considerablemente menores a lo reportado para otras especies de psitácidos (Alvarado 2008; Soto 2009) inclusive por lo reportado por Santos (1999) para Amazona aestiva aestiva. Los heterófilos estuvieron por encima de lo que se ha reportado 
en estudios previos en psitaciformes (Polo et al. 1998; Santos 1999). La heterofilia puede atribuirse parcialmente a la excitación y liberación de adrenalina, que causaría la redistribución de heterófilos marginales hacia la circulación central. El estrés genera liberación de corticosteroides que se asocia a leucocitosis, neutrofilia/heterofilia, eosinopenia y linfopenia (Dunn 2000). Polo et al. (1998) utilizaron Isoflurano para la colecta de sangre mientras que para el presente estudio sujeción física, por lo que la excitación durante ambos eventos fue diferente y pudo influir en los resultados. Los leucocitos más abundantes en aves son tanto linfocitos como heterófilos y esto ha sido descrito en otros psitácidos (Polo et al. 1998).

A pesar de que por regla general hay más linfocitos que heterófilos en aves, estudios previos de Polo et al en falconiformes (1992), columbiformes (1992) y otros (citados por Polo et al. 1998) marcan tendencias hacia la heterofilia y otros autores también han descrito esta tendencia en aves. A pesar de esto, la diferencia observada pudo deberse más al error humano en la interpretación de leucocitos que a la influencia marcada por el taxón del ave. Otros factores que pueden alterar los resultados (de hematología o química) son la variación del hábitat, estado fisiológico, edad, nutrición o el uso de anestésicos (Mader 2006).

No se encontraron diferencias significativas para ninguno de los parámetros en estudio entre ambos sexos (cuadro 2). Los valores son presentados como la media, intervalos de confianza al $95 \%$ y significancia (p) en el cuadro 2.

Cuadro 2. Comparación por sexo de valores hematológicos para Amazona aestiva xanthopteryx, cautivos en la Estación de Rescate, Rehabilitación y Reubicación de Fauna "La Esmeralda” de la Provincia de Santa Fe en Argentina.

\begin{tabular}{llccc}
\hline & & $\begin{array}{c}\text { Machos } \\
N=15\end{array}$ & $\begin{array}{c}\text { Hembras } \\
N=12\end{array}$ & $\mathrm{p}$ \\
& & Media \pm I.C. 95\% & Media \pm I.C. 95\% & \\
\hline Eritrocitos & $(1.00 \mathrm{E}+06)$ & $3.06 \pm 0.388$ & $3.01 \pm 0.275$ & 0.3 \\
VCM & $(\mathrm{fL})$ & $157.41 \pm 27.811$ & $162.03 \pm 18.015$ & 0.4 \\
HCM & $(\mathrm{Pg})$ & $53.95 \pm 5.989$ & $53.06 \pm 3.955$ & 0.3 \\
CHCM & $(\mathrm{g} / \mathrm{dl})$ & $29.98 \pm 3.823$ & $30.810 \pm 3.650$ & 0.4 \\
Leucocitos & $(1.00 \mathrm{E}+03)$ & $6.57 \pm 4.102$ & $5.99 \pm 2.355$ & 0.4 \\
Heterófilos & $(\%)$ & $78.13 \pm 14.478$ & $76.75 \pm 11.417$ & 0.7 \\
Linfocitos & $(\%)$ & $17.83 \pm 14.640$ & $19.50 \pm 12.352$ & 0.3 \\
Monocitos & $(\%)$ & $2.33 \pm 3.378$ & $2.75 \pm 4.537$ & 0.9 \\
Eosinófilos & $(\%)$ & $0.79 \pm 2.020$ & $0.38 \pm 1.458$ & 0.1 \\
Basófilos & $(\%)$ & $0.24 \pm 0.886$ & $0.63 \pm 1.458$ & 0.2 \\
Plaquetas & $(1.00 \mathrm{E}+03)$ & $24.58 \pm 30.283$ & $24.46 \pm 34.012$ & 0.5 \\
\hline
\end{tabular}

Aunque no se observaron diferencias significativas entre machos y hembras, los datos deben tratarse con reserva dado que las aves no estaban en estación reproductiva al momento del muestreo y la influencia hormonal sobre los valores evaluados pudo no ser observada. 
Los loros habladores del chaco se reproducen entre octubre y marzo (Narosky \& Yzurieta 2010). La ausencia de efecto del sexo sobre hematología que se observó ha sido reportada en otras especies monomórficas, pero en contraposición, se ha observado cierta influencia del sexo sobre este tipo de valores en especies dimórficas como el faisán común Phasianus colchicus; (Hauptmanova et al. 2006) que durante la estación reproductiva la influencia hormonal afectó algunos valores, principalmente en los machos.

\section{Conclusiones}

En general, los resultados obtenidos para la subespecie Amazona aestiva xanthopteryx, fueron similares a los reportados en la literatura para otras aves del mismo orden, mientras que en comparación con Amazona aestiva aestiva los valores de Hematocrito (Hto), Hemoglobina $(\mathrm{Hb})$ y heterófilos fueron mayores en Amazona aestiva xanthopteryx, pero menor fue el recuento de linfocitos para ésta última. Esto puede deberse a las distintas condiciones geográficas, ambientales y de manejo entre los diferentes estudios.

Se destaca que éste trabajo es uno de los primeros en Argentina en ofrecer datos sobre valores sanguíneos de Amazona aestiva xanthopteryx, por lo que los mismos ayudarán a la toma de decisiones en los protocolos de manejo para dicha subespecie.

\section{Agradecimientos}

Agradecemos al personal de Estación Biológica La Esmeralda (Santa Fe, Argentina) y a la Facultad de Ciencias Veterinarias de la Universidad Nacional del Litoral (FCV-UNL, Argentina) por la orientación y asistencia durante el estudio.

\section{Declaración de intereses}

Los autores no informaron ningún conflicto de intereses potencial.

\section{Referencias}

Alvarado M.C., Arraga-Alvarado C., Rincón M., Fernández G., Aguilar J., Villasmil-Ontiveros Y., Gómez O., \& Henríquez A. 2008. Valores hematológicos de psitácidos de los géneros Ara y Amazona cautivos en zoológicos de Venezuela. Revista Científica, FCV-LUZ/ Vol. XVIII, No 6, 649-661. http://redalyc.uaemex.mx/src/inicio/ArtPdfRed.jsp?iCve=95911659002

Banchs, R., Moschione, F., \& Vaca, R. 2006. Resultados del trabajo del "Proyecto Elé" de uso sustentable del loro hablador Amazona aestiva (Aves: Psitaciformes) durante el período 1997-2004. Resumen de las experiencias. 1 APRONA Bol. Cient. 39: 1-11.

Berkunsky, I., Quillfeldt, P., Brightsmith, D.J., Abbud, M.C., Aguilar, J.M.R.E., Alemán-Zelaya, U., Aramburú, R.M., Arce Arias, A., Balas, M.N., Balsby, T.J.S., Barredo Barberena, J.M., Beissinger, S.R., Rosales, M., Berg, K.S., Bianchi, C.A., Blanco, E., Bodrati, A., Bonilla-Ruz, C., \& Masello, 
J.F. 2017. Current threats faced by Neotropical parrot populations. Biol. Conserv. 214: 278-287. Doi: 10.1016/j.biocon.2017.08.016

Chaves Cordeiro, P.H. 2004. A Fragmentação da Mata Atlântica no Sul da Bahia e suas implicações na conservação dos psitacídeos. In Prado, P.I., Landau, E.C., Moura, R.T., Pinto, L.P.S., Fonseca, G.A.B., Alger, K.N. Corredor de Biodiversidade da Mata Atlântica do Sul da Bahia. Publicação em CD-ROM, Ilhéus.

Chébez, J.C. 2008. Los que se van Tomo 1: Problemática ambiental, Anfibios y Reptiles. Albatros, Buenos Aires. p. 320

Convention on International Trade in Endangered Species of Wild Fauna and Flora (CITES). 2017. Apéndices I, II y III. https://www.cites.org/sites/default/files/esp/app/2013/SAppendices-2013-06-12.pdf Acceso: 12 de febrero de 2019.

Deem, S.L., Noss, A.J., Cuéllar, R.L., \& Karesh, W.B. 2005. Health evaluation of free ranging and captive blue-fronted amazon parrots (Amazona aestiva) in the Gran Chaco, Bolivia. J. Zoo Wildl. Med. 36(4): 598-605. Doi: 10.1638/04094.1

Dunn, J. 2000. Disorders of leukocyte number. Manual of canine and feline haematology and transfusion medicine. In Day, M.J., Mackin, A., \& Littlewood, J. (Eds). British Small Animal Veterinary Association, Glouchester. p. 93-105.

Franco, M., Hoyos, L., Ramírez, G.F., \& Correa, A.M. 2010. Hallazgos hematológicos y química sanguínea en Amazona amazonica y Amazona ochrocephala cautivas de la reserva forestal torre cuatro. Bol. Cient. Mus. Hist. Nat. 13(2): 63-77.

Hauptmanova, K; Maly, M., \& Literak, I. 2006. Changes of haematological parameters in common pheasant (Phasianus colchicus) throughout the year. Veterinarni Medicina 51 (1): 29-34.

Ike, S.O., Nubila, T., Ukaejiofo, E., Nubila, I.N., Shu, E.N, \& Ezema, I. 2010. Comparison of haematological parameters determined by the Sysmex KX-2IN automated haematology analyzer and the manual counts. BMC Clin. Pathol. 10(3): 1-5. Doi: 10.1186/1472-6890-10-3.

Mader, D.R (ed). 2006. Reptile Medicine and Surgery, 2nd Edition. Saunders Elsevier, Missouri. p. 1242

Meneses, A., Villalobos, J. \& Sancho, E. 1993. Manual de hematología y química clínica en medicina veterinaria. Fundación UNA, Heredia. p. 168.

Mérida Ruiz, S.A. \& Guerra Centeno, D.S. 2011. Valores preliminares de referencia para hematología y química sérica del loro frente roja (Amazona autumnalis) en cautiverio. REDVET 12(3): 1-11.

Naidoo, V., Diekmann, M., Wolters, K. \& Swan, G.E. 2008. Establishment of selected baseline blood chemistry and hematologic parameters in captive wild-caught African white-backed vultures (Gyps africanus). J. Wildlife Dis. 44(3): 649-654. Doi: 10.7589/0090-3558-44.3.649

Narosky, T., \& Yzurieta, D. 2010. Aves de Argentina y Uruguay: Guía de identificación, 16ava Edición. Vázquez Mazzini Editores, Buenos Aires. p. 432. 
Paula, V.V., Fantoni, D.T., Otsuki, D.A. \& Auler Jr, J.O.C. 2008. Blood-gas and electrolyte values for Amazon parrots (Amazona aestiva). Pesq. Vet. Bras. 28(2):108-112. Doi: 10.1590/S0100736X2008000200003.

Polo, F.J.; Peinado, V.I.; Viscor, G. \& Palomeque, J. 1998. Hematologic and plasma chemistry values in captive psittacine birds. Avian Diseases 42: 523-535. DOI: 10.2307/1592679

Santos, L.C. 1999. Laboratorio ambiental. Editora Universitária Edunioeste, Cascavel. p. 341. ISBN: 8586571-29-6 http://www.unioeste.br/editora/lista trab.asp?codvalue=85-86571-29-6

Schmidt, E.M., Lange, R.R., Ribas, J.M., Daciuk, B.M., Montiani-Ferreira, F., \& Paulillo, A.C. 2009. Hematology of the Red-capped parrot (Pionopsitta pileata) and Vinaceous Amazon parrot (Amazona vinacea) in captivity. J. Zoo Wildl. Med. 40(1): 15-17. Doi: 10.1638/2007-0054.1

Sokal, R. \& Rohlf, J. 1995. Biometry: The principles and practice of statistics in biological research, 3rd Edition. Freeman and Company, New York. p. 887.

Soto Piñeiro, C.J., Acosta Guevara, I. \& Cruz López, E. 2009. Parámetros hematológicos de Cotorras (Amazona leucocephala) y Cateyes (Aratinga euops). REDVET 10(7B): 1-12.

Valle, S. de F., Allgayer, M. da C., Pereira, R. A., Barcellos, L.J.G., Hlavac N.R.C., Franca, R.T., \& Locateli, M.L. 2008. Parâmetros de bioquímica sérica de machos, fêmeas e filotes de Araras canindé (Ara ararauna) saudáveis mantidas em cativeiro comercial. Ciênc. Rural 38(3): 711-716. Doi: 10.1590/ S0103-84782008000300018 